\title{
Design and Analysis of Non-Conventional Type Cigarette Automatic Palletizing Tray Institutions
}

\author{
Weimin Lin $^{1,}{ }^{*}$, Kangqi $\mathrm{Mu}^{2}$, Dong Sun ${ }^{3}$, Ping Zuo ${ }^{4}$ \\ ${ }^{1}$ Key Laboratory of Advanced Manufacturing technology, Ministry of Education, Guizhou University, \\ Guiyang, China; \\ ${ }^{2}$ School of Mechanical Engineering, Guizhou University, Guiyang, China; \\ ${ }^{3}$ Guizhou Institute of Technology, Guizhou University, Guiyang, China; \\ ${ }^{4}$ Guizhou Tobacco Companies Bijie Company, Bijie, China. \\ *361108958@qq.com
}

Keywords: Non-conventional type cigarette; automatic palletizing; tray.

\begin{abstract}
Aiming to solute low efficiency in sorting and packaging process of non-conventional type cigarette, a steady and rapid automatic palletizing tray institution was designed in this paper. Based on the three-dimensional model of the institution established by software of SolidWorks, the mechanical properties of the institution were analyzed. The results showed that the most obvious deformation occurred on the top right-hand corner of pushing board, but it does not affect the actual work effect of pushing cigarette mechanism. The largest stress point is on connection of the pushing rod, and the value of max stress is less than the material yield strength. The designed institution is safe. Furthermore, production practice showed that the designed tray body performed with high efficiency and stability. The efficiency reached 7300 bar/hour. It showed that the designed palletizing tray institutions is reasonable and feasible.
\end{abstract}

\section{Introduction}

Non-conventional type cigarette refers to the packaging specification is different from the standard cigarette. It is difficult to be sorted and packaged automatically as standard cigarette. In practice, nonconventional type cigarette is often sorted by manual work, which cost intensive labor, work in low efficiency and high cost. This becomes an urgent problem need to be solved in non-conventional palletizing work.

In 2017, annual sales of non-conventional type cigarette were up by $93.0 \%$ year-on-year. The sales tell us a good market development of the items, and gradually become a new driving force of economic growth to relative enterprises. Launching non-conventional type cigarette automatic sorting and palletizing system, as well as developing new packaging equipment, has a broad application prospect to meet the demand of non-conventional type cigarette logistics [1].

At present, cigarette automatic sorting and palletizing system is mainly applied in standard cigarette logistics, and the technology is well developed, but little work has been done on nonconventional type cigarette. Most of tobacco logistics companies deal non-conventional type cigarette with manual work. Therefore, we designed a kind of automatic palletizing tray institutions to provide technical support for the development of non-conventional type cigarette automatic sorting and palletizing system.

\section{Design of the Palletizing Tray Institution}

At present, automatic palletizing is widely used in tobacco logistics companies, the main way of non-conventional type cigarette automatic palletizing is operated by manipulator. While, the height and capacity of cigarette stack are limited by the vertical working distance of manipulator. In addition, the stack should be conveyed smoothly to the next station after palletized to guarantee the work efficiency of palletizing system. In this process, the relative displacement among cigarettes should be 
controlled I within allowable limits. Based on above requirements, we designed an elevating palletizing tray institution for non-conventional type cigarette.

Compared with the common palletizing way on the market, the designed institution has several advantages, including:

(1) Pallets can go down as the growth of the height of cigarette stack step by step. It greatly improves the upper height limit of the cigarette stack and increases the capacity of each cigarette stack.

(2) The institution can divide the belt into two layers, and drastically reduce the occupied area of equipment and save the space.

(3) Bilateral palletizing can be adopted in the system. The standby time of manipulator can be effectively shortened and significantly improved the efficiency of palletizing.

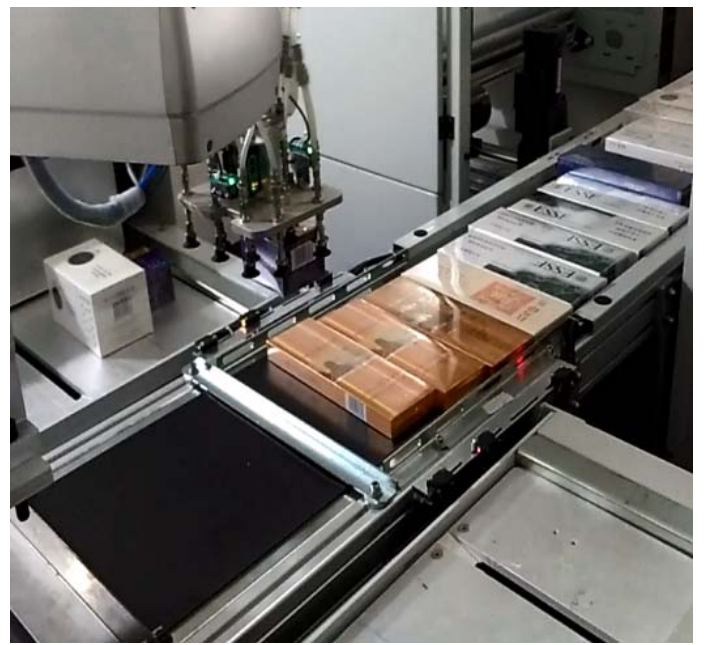

Figure 1. Stacking pallet body

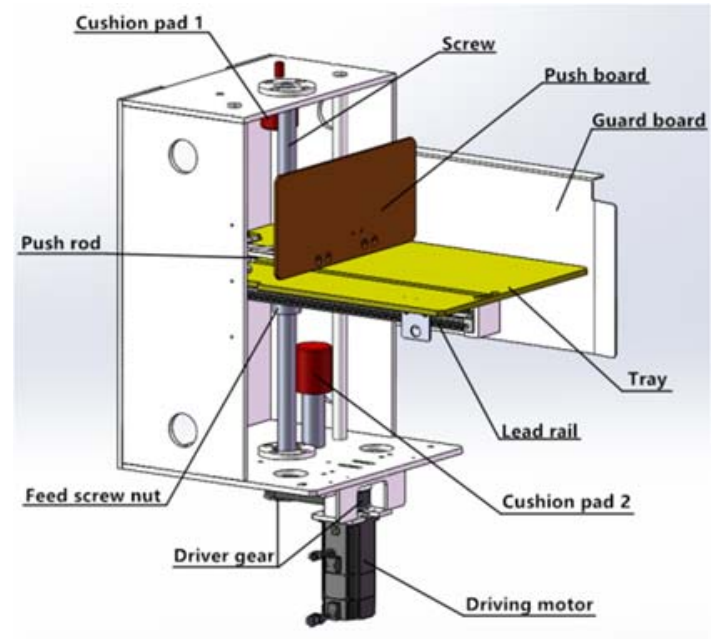

Figure 2. Stacking pallet body model

As shown in Fig.2, the elevating palletizing tray institution for non-conventional type cigarette is constructed by spiral screw lift platform and push plate. The main functions of this institution are to receive the cigarette stack put on the platform by manipulator and transport the stack to the belt for the next station in the working system.

During the institution is working, each motion of elevating platform and push plate will bring oscillation to the pallet, the tray will suffer from the stress deliver by the motion, and deformation is likely to be occurred. Furthermore, the stability of non-conventional type cigarette stack is worse than that of standard cigarette, thus the deviation or collapse is likely easy to occur, which will affect the 
system to serve continuously. It means the stability of the pushing cigarette board is an important factor to ensure the quality of the tray institution.

Palletizing tray institution is the most important basic part of the non-conventional type cigarette sorting system. Its performance will directly affect the work efficiency and stability of the sorting system, so we need to carry out static analysis on the body using the finite element analysis software.

\section{Finite Element Analysis}

In the entire tray device, pushing cigarette mechanism is the key part which receives great press and impact, so it is most likely to be damaged. Furthermore, pushing cigarette mechanism contacts with cigarette stack directly, the deformation of this part may damage cigarette stack type and affect the efficiency of system. 3D model of pushing cigarette mechanism is set up by software SolidWorks before carrying out finite element analysis.

In order to guarantee the accuracy of calculation and economize computational cost, the model is simplified. We remove small screw holes, chamfer angle, circular bead, remove convex platform in the non-critical parts, and simplify the structure of internal parts and rolling guide block [2, 3]. Otherwise, it will greatly increase the number of grid, extend the time of calculation, and even led to the incorrect results or unable to converge $[4,5]$.

After removing some small chamfer angle and small holes unlikely affect system assembly, we divide pushing cigarette mechanism into a lot of high quality hybrid grids. The cell size is 13.0705 $\mathrm{m}$, the tolerance is 0.6535 , the number of total section is 52488 , and the number of total cell is 30182 . The grid pattern is shown in Fig.3.

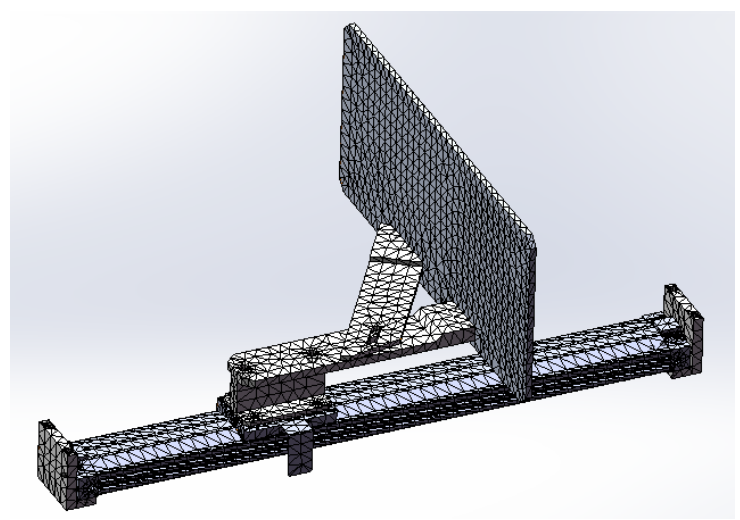

Figure 3. Pushing cigarettes mechanism grid

\section{Static Analysis}

Static stiffness means the ability of mechanism to resist deformation of pushing cigarette mechanism under static load conditions [6]. Pushing cigarette mechanism is designed as an elastic system. Because mechanical deformation of the institution will be produced under the thrust from cylinder and the counterforce from cigarette stack, as well deformation also can be occurred by contact between the push board and cigarette. All the deformation will lead to the relative displacement between pushing plate and the cigarette stack; finally affect the stability of the stack. So, the main part of pushing cigarette mechanism needs enough stiffness to reduce the effects caused by deformation on the stability of cigarette stack.

The thrust of pushing cigarette mechanism in horizontal direction is mainly made up of the thrust from cylinder and the counter force from cigarette stack. According to period of pushing motion and working distance,

$$
\mathrm{s}=\frac{1}{2} \mathrm{at}^{2}
$$

The acceleration of pushing cigarette plate can be calculated.

The stress analysis for the thrust of pushing cigarette mechanism in horizontal direction is shown in Fig. 4. 


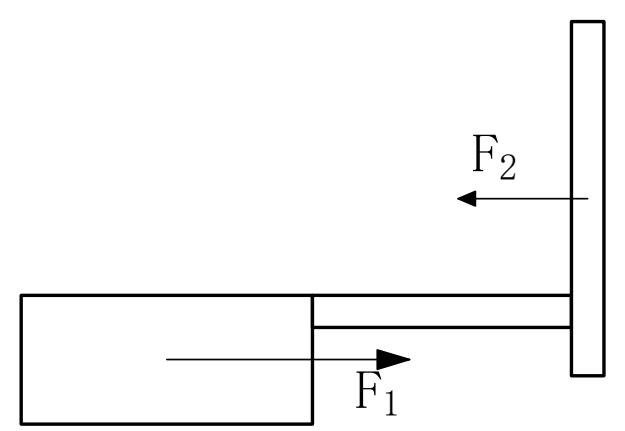

Figure 4. The stress analysis diagram

$$
\begin{aligned}
& \mathrm{F}_{\mathrm{x}}=\mathrm{ma} \\
& \mathrm{F}_{\mathrm{x}}=\mathrm{F}_{1}-\mathrm{F}_{2}
\end{aligned}
$$

Using the simulation module, the stress in the model is analyzed. We assume that the model is made up from $45 \#$ steel material. The result shows that its elasticity modulus is $2.05 \times 1011 \mathrm{~N} / \mathrm{m} 2$, Poisson's ratio is 0.29 , mass density is $7850 \mathrm{~kg} / \mathrm{m} 3$ and the yield strength is $5.3 \times 108 \mathrm{~N} / \mathrm{m} 2$. We define both the cylinder blocks as the fixed parts. According to the actual working situation, define the interaction between parts and force the external load.

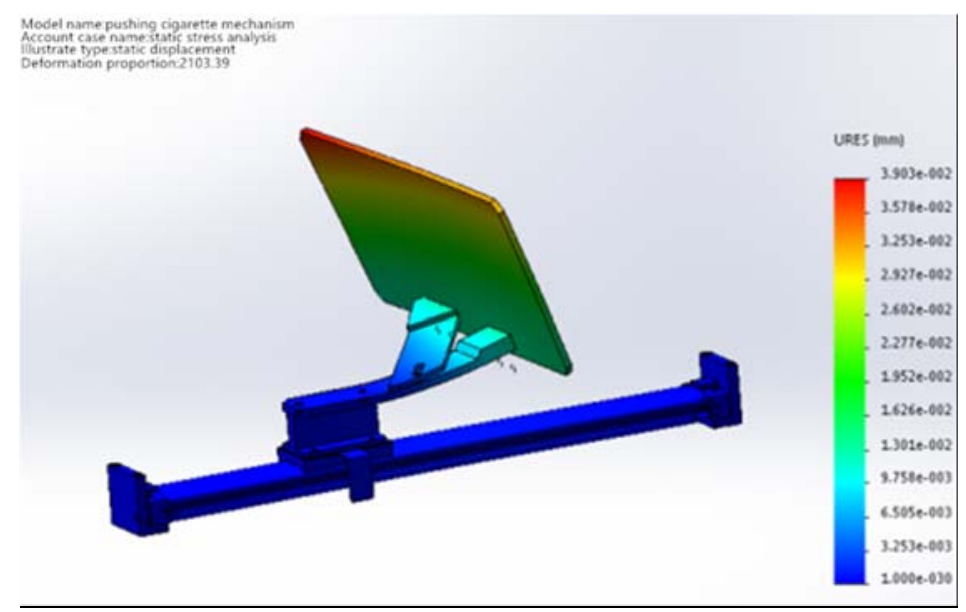

Figure 5. Displacement distribution of pushing cigarette mechanism

According to the resultant displacement distribution cloud image (Fig.5), we find the main way of deformation on pushing cigarette mechanism is originated from the pushing plate and the push rod part become warped up, and mainly caused by the bending deformation in the joint of push rod. The most obvious deformation occurred on the top right-hand corner of pushing board. The deformation quantity is $3.903 \mathrm{e}-02 \mathrm{~mm}$. Therefore, it may cause the stack tilted slightly, but the quantity does not affect the actual work effect of pushing cigarette mechanism.

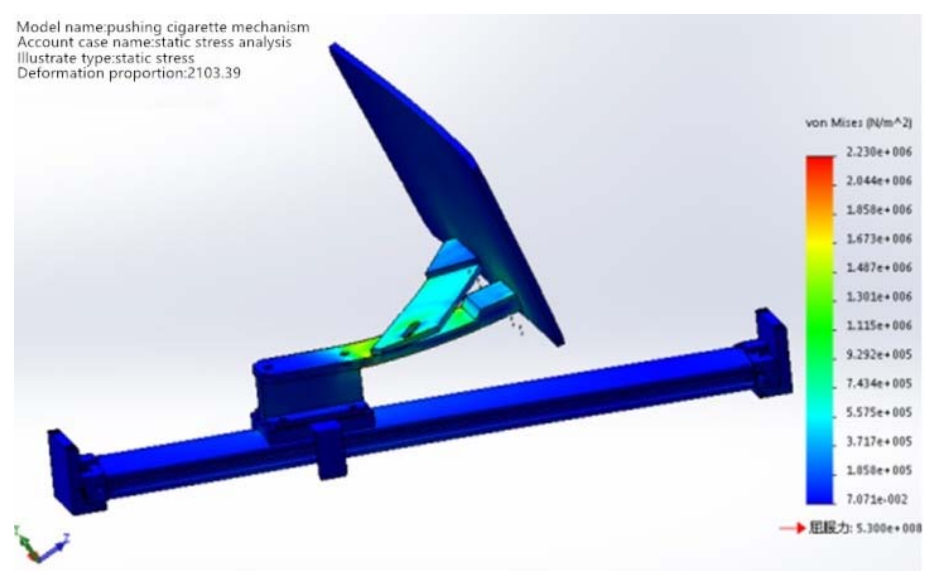

Figure 6. Stress distribution of pushing cigarette mechanism 
According to the stress distribution of pushing cigarette mechanism (Fig.6), the stress on the whole mechanism is not so large and can be beard on most places. The largest stress point is on connection of the pushing rod. The max stress is $2.23 \times 106 \mathrm{~N} / \mathrm{m} 2$, which is less than the material yield strength. It indicates material damage can be avoided. It will guarantee the work effect of pushing cigarette mechanism.

\section{Application Effect}

The designed institution has been applied in several tobacco logistics centers, such as Tobacco Companies in Wenzhou city of Zhejiang province, Defang city of Sichuan province, and Benjie city of Guizhou province. The equipment is shown in Fig.7.

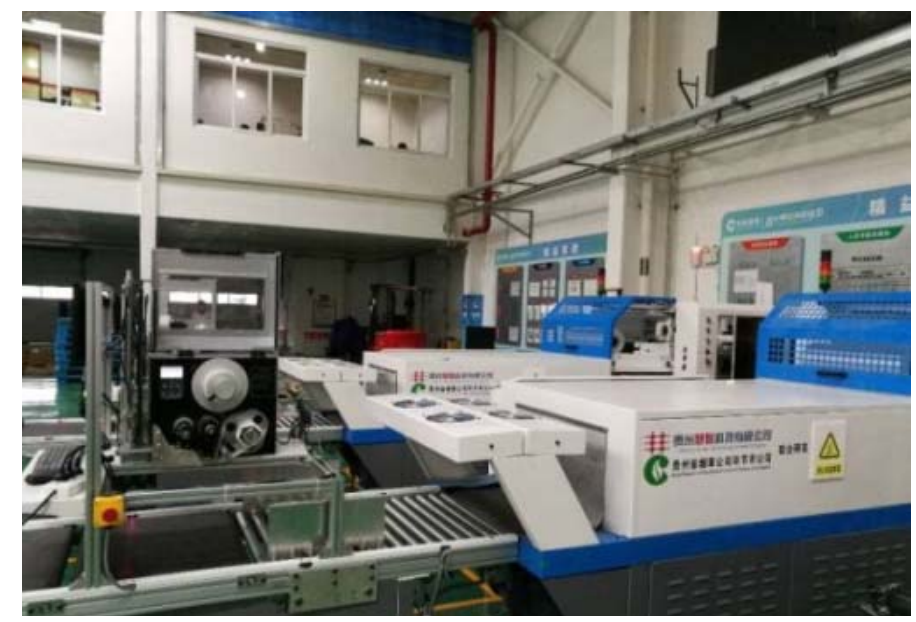

Figure 7. Non-conventional type stacking and packing equipment

In Benjie Company of Guizhou tobacco Companies, we tested the functions of the institution in non-conventional type stacking and packing equipment. The working efficiency data were collected randomly for 10 times (Tab.1). The results showed that the working efficiency of the machine is stable, working efficiency (except downtime) reaching 7300 bars per hour.

Table 1. sorting efficiency statistical table

\begin{tabular}{cccc}
\hline No. & $\begin{array}{c}\text { Total number } \\
\text { (bar) }\end{array}$ & $\begin{array}{c}\text { Working efficiency (except downtime) } \\
\text { (bar/hour) }\end{array}$ & $\begin{array}{c}\text { Combined efficiency (contain downtime) } \\
\text { (bar/hour) }\end{array}$ \\
\hline 1 & 12336 & 7242 & 5952 \\
2 & 10319 & 7330 & 5965 \\
3 & 10187 & 7279 & 6345 \\
4 & 8496 & 7376 & 6575 \\
5 & 6035 & 7310 & 6893 \\
6 & 4647 & 7487 & 7087 \\
7 & 2282 & 7164 & 7786 \\
8 & 1347 & 7238 & 7771 \\
9 & 916 & 7290 & 7091 \\
10 & 181 & 7945 & 7586 \\
\hline
\end{tabular}

*Note: downtime means the pause caused the shift of workers, the low efficiency of workers, the breakdown of the machine and so on.

\section{Conclusion}

Design of non-conventional type cigarette automatic palletizing tray institutions adopts pneumatic drive mechanism and the ball screw lift platform. The institution improves the allowed height of cigarette stack and the efficiency of the system. It ensures cigarette stack transport smoothly and quickly from the tray into the lower belt for packaging. The average efficiency reaches 7300 bar per hour. Equipment adopts modular design, so that it's convenient to be operated and maintained. It takes an important part in non-conventional type cigarette system. In Benjie, Defang and Wenzhou 
city, the mechanism works efficiently and stably. This research has important significance for improving the efficiency of non-conventional type cigarette sorting.

\section{Acknowledgments}

This work was financially supported by Guizhou Province Science and Technology Project (Agricultural Machinery Intelligent Instrumentation Technology Research and Industrialization, Grant No. [2017]2308) and Guiyang National Hi-Tech Industrial Development Zone, high-level talent innovation and business projects (Research and Industrialization of Intelligent Shaped Cigarette Sorting and Collecting System) and Guizhou Province, industrial and information technology development(Research and development and Industrialization of Intelligent Shaped Object Sorting System, Grant No.2017039).

\section{References}

[1]. Changing Ling. Insist on maintaining stability tone always strive for stability in the new positive as "two super trillions of" the excellent result to meet the victory at the party's $19[\mathrm{~N}]$. Oriental tobacco newspaper, Jinan, 2017-01-17(002).

[2]. Dong Sun, Ping Zhang, Xuedong Yang. Analysis and research of XK2430 beam structure [J]. Modern Machinery, Guiyang, 2015(3):56-58.

[3]. Zhou Zhuang. Analysis and application based on ABAQUS finite element [M]. Tsinghua University Press, Beijing, 2009: 151-369.

[4]. Wang Goo, Chao Hu. Dynamics of cantilever plates and its vibration control [J]. Journal of Dynamics and Control, Changsha, 2010, 8(1):80-86.

[5]. Shading Zhang. Introduction to the development and application of computer aided engineering (CAE) [J]. Public Communication of Science \& Technology, Beijing, 2010(16): 232-233.

[6]. Xuedong Yang, Dong Sun, Qian Yi, etc. Weight-reduction Optimization Design for Milling Planer Bed Based on Finite Element Analysis [J]. Machine Tool \& Hydraulics, Guangzhou, 2013, 41(4):17-20. 